\title{
Anticipatory care in very deprived areas
}

A paradox of public health practice within the NHS is that while doctors with public health in their job titles tend to have little contact with the public, GPs who have substantial contact with the public tend not to think about public health. Both groups tend to underestimate the public health function of general practice.

When 'Willie' Sutton, the famous US bank robber, was asked why he robbed banks, he replied, 'Because that is where the money is'. When asked why general practice is important for public health, the answer is, 'Because that is where the contact is'.

Serial contacts with individual patients in general practice provide continuity, flexibility, shared knowledge, long-term relationships and trust - all key ingredients of effective long-term care. Cumulative contact with practice patients provides a large measure of population coverage. Whereas screening and research are considered to have done well when they reach $70 \%$ of the population, primary care regularly has contact with over $90 \%$. No other part of the NHS has this degree or type of coverage.

The contact is of a particular kind, however, contrasting with the cold-calling approach of screening programmes and is almost always instigated by patients, requiring practitioners to address the presenting problem before moving on, if appropriate, to other issues.

The pioneer of anticipatory care in general practice was Julian Tudor Hart. ${ }^{1}$ Although his example has been used by several Ministers of Heath to justify programmes of cardiovascular risk screening via health checks, Tudor Hart never screened his practice population and never used health checks. Instead, he used routine contacts to build up coverage of his population, using special measures only for patients who had been missed out using this approach.

The other major misperception of his work is that the approach was not restricted to applying the evidence-based medicine of the day (anticipating the Quality and Outcomes Framework), mainly addressing the risks of high blood pressure, smoking and diabetes. The cardinal feature of his practice was an unconditional approach to all of the problems presented by his patients, providing continuity and coordination, and using epidemiology to measure what he had and hadn't done. The epidemiology was new, but the interventions were mainstream.

As part of the Deep End Project, 20 GPs from Glasgow, Edinburgh and Inverclyde met to review their experience of Keep Well, the Scottish national anticipatory care programme, including cardiovascular health checks and referral to health improvement programmes including smoking cessation, exercise, obesity and other health

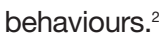

The group concluded that focusing on the 45-64-year age group was starting too late in deprived areas, where people aged 5564 years are already 'older' in terms of reduced life expectancy and the prevalence of multiple morbidity. Deep End practitioners know many patients in their 30 s with multiple risks and problems who would benefit from anticipatory care.

While screening programmes can process large numbers of people very quickly, they lack sustainability, are generally poor at contacting the last $30 \%$ of the population and impose an external agenda, which may be neither sufficient nor timely, especially for patients with multiple problems.

The health check approach 'of encouraging patients to attend for a health check and then to support them to follow an agreed behaviour change and or treatment regime' is simplistic and flawed, in relation to the needs of patients with complex needs. The experience of Deep End GPs is that while health checks have worked for some patients, there are many patients, especially those with alcohol, literacy, psychological and social problems, for whom health checks work less well, and whose involvement in health improvement activities requires a different approach. The problems of alcohol are particularly acute, and a greater cause of premature death in severely deprived areas than cardiovascular disease. In general, increased case-loads for

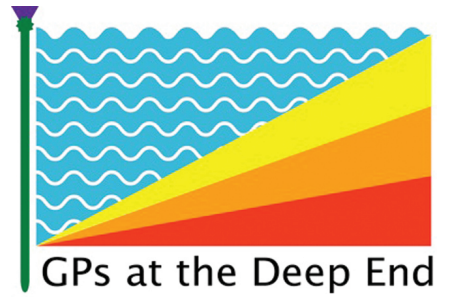

cardiovascular risk prevention in general practice, as generated by screening programmes, are only feasible with additional resources, a team approach and effective links to external professions and services. Such external resources are much more plentiful than they were in Tudor Hart's day, but are often not effectively integrated with the opportunities and needs for health advice and support, occurring regularly but fleetingly in day-to-day practice.

The best arrangements for supporting practices serving areas of blanket deprivation are unlikely to be the same as those required by practices serving areas of pocket deprivation. In Scotland, 50\% of people living in very deprived areas (the most deprived $15 \%$ of postcodes) are registered with the 100 Deep End practices. The other $50 \%$ are registered with 700 other practices in Scotland. As 'Willie' Sutton might have said, resources need to be concentrated 'where the deprivation is'.

Like compound interest, the public health benefits of mainstream general practice accrue not immediately but towards the end of a period of sustained investment. ${ }^{3}$ The challenge is not only to ascertain risks, but also to follow this up, working with patients (in Tudor Hart's phrase 'initially face to face, eventually side by side'), flexibly but consistently, as needs determine, over the long term. By the sum of such relationships, the NHS can improve health and narrow inequalities.

\section{Graham Watt}

On behalf of the Deep End Steering Group. This is the third of 12 articles from GPs at the Deep End.

\section{REFERENCES}

1. Watt G O'Donnell C Sridharan S. Building on Julian Tudor Hart's example of anticipatory care. Prim Health Care Res Dev 2011; 12(1): 3-10.

2. Deep End Report 4. Experience and views of Keep Well and ASSIGN. The fourth meeting of 'General Practitioners at the Deep End', 29 Jan 2010.

http://www.gla.ac.uk/media/media_146573_en.pdf (accessed 3 Feb 2011).

3. Hart JT, Thomas C, Gibbons B, et al. Twenty five years of case-finding and audit in a socially-deprived community. BMJ 1991; 302: 1509-1513

DOI: 10.3399/bjgp11X561401 